\title{
Non-localized acoustic receptivity and subsequent disturbance growth in a Blasius boundary layer
}

\author{
Rudolph A. King ${ }^{1}$ and Kenneth S. Breuer ${ }^{2}$ \\ 1 NASA Langley Research Center, Hampton VA 23681, USA \\ 2 Division of Engineering, Brown University, Providence RI 02912, USA
}

\begin{abstract}
Acoustic receptivity was experimentally examined for a Blasius boundary layer with receptivity sites in the form of 2-D waviness and oblique waviness. Linear receptivity coefficients obtained for all values of forcing combinations $(\epsilon \cdot \Delta h)$ are in good agreement with receptivity theory. The measured receptivity increased markedly with increasing wave obliqueness. Detuning was investigated by varying the streamwise wavenumber, $\alpha_{w}$, of the $2-\mathrm{D}$ and oblique wall waviness. Oblique transition was also investigated by exciting a pair of oblique Orr-Sommerfeld $(\mathrm{O}-\mathrm{S})$ modes $\left(f / f_{o}, \pm \beta / \beta_{w}\right)=(1, \pm 1)$. For large values of $\epsilon \cdot \Delta h$, a secondary instability was observed that ultimately lead to laminar breakdown. This instability appears to be driven by large spanwise velocity gradients, $|d U / d \zeta|$.
\end{abstract}

\section{Introduction}

The process by which freestream disturbances are internalized to generate boundary-layer instability waves is referred to as receptivity. A fundamental understanding of receptivity is important since it provides the initial conditions for the subsequent growth of the unstable modes which may lead to laminar breakdown. Because of the significant wavelength mismatch between the long-wavelength external disturbances and the much shorter wavelength Tollmien-Schlichting (T-S) waves, a length-scale conversion must take place to facilitate the energy transfer. It has been shown [1-4] that wavelengthreduction mechanisms can range from direct scattering associated with abrupt changes in surface conditions to the gradual wavelength reduction due to viscous boundary-layer growth.

A transition mechanism, the so-called oblique transition, involving a pair of oppositely-oriented oblique $\mathrm{O}-\mathrm{S}$ modes $(1, \pm 1)$ was first recognized using direct numerical simulations applied to a channel flow [5]. This transition scenario was conjectured as a three stage process: $(i)$ a nonlinear interaction of primary disturbances to generate a streamwise vortex system $(0, \pm 2),($ ii $)$ a transient growth region of low- and high-speed streaks produced by the vortex system, and (iii) ultimate breakdown of the steaks instigated by nonstationary disturbances due to secondary instability. Oblique transition was later verified in a physical experiment conducted in a plane Poiseuille flow [6].

In this experimental study, acoustic receptivity was examined for a Blasius boundary layer. Non-localized surface roughnesses, 2-D and oblique, were considered for various roughness heights, $\Delta h$, and acoustic wave amplitudes, $u_{a c}\left(\epsilon=u_{a c} / U_{\infty}\right)$. A plane downstream-traveling acoustic wave was created to excite the flow near the resonance frequency, $f_{0}$, of an unstable $\mathrm{O}-\mathrm{S}$ eigenmode 
corresponding to the roughness wavenumber $k_{w}=k_{t s}$. Branch I was selected to occur near the streamwise center of the roughness to maximize the receptivity. Oblique transition was also investigated by exciting a pair of oblique $\mathrm{O}-\mathrm{S}$ modes $\left(f / f_{o}, \pm \beta / \beta_{w}\right)=(1, \pm 1)$. Further details of the experimental study are given in Reference [7].

\section{Experimental apparatus and procedure}

The experiment was conducted in the 2 Foot by 3 Foot Low-Speed Wind Tunnel located at NASA Langley Research Center. Turbulence intensities, $u / U_{\infty}$, of $0.1 \%(0.1<f<400 \mathrm{~Hz})$ were measured. The experiment was conducted with a nominal freestream velocity $U_{\infty}=11.1 \mathrm{~m} / \mathrm{s}$. The model was a 12.7-mm-thick flat aluminum jig plate with a $24: 1$ elliptical leading edge and a $0.2-\mu m$ surface finish on the test side. The receptivity sites were manufactured with copper-plated circuit boards having three distinct copper thicknesses $\Delta h_{1}=17.8 \mu \mathrm{m}, \Delta h_{2}=2 \Delta h_{1}$ and $\Delta h_{3}=4 \Delta h_{1}$. The patterns were generated using a photolithographic process which insured high spatial accuracy. A twolayer pattern was produced with $\Delta h$ being equal to the copper thickness. At Branch I for $\Delta h_{3}$, the roughness Reynolds number was $R e_{\Delta h} \approx 47$, within the hydraulically smooth limit. Upstream and downstream speakers were used to generate the desired acoustic field [4]. The acoustic wave train was excited at $f_{0}=71 \mathrm{~Hz}\left(F=55 \times 10^{-6}\right)$. The acoustic levels examined were $\epsilon_{1}=7.6 \times 10^{-5}$, $\epsilon_{2} \doteq 2 \epsilon_{1}$, and $\epsilon_{3} \doteq 4 \epsilon_{1}$.

A single hot-wire probe was utilized to measure the streamwise velocity. $\mathrm{T}-\mathrm{S}$ wave extraction involved two profile measurements, one with the smooth surface (included the Stokes wave, T-S wave due to leading-edge receptivity, and extraneous disturbances), and the other with the surface roughness (included same components as smooth surface plus T-S wave due to roughness). The T-S wave due to roughness was obtained by subtracting in the complex plane the smooth surface profiles from the rough surface profiles.

\section{Results and discussion}

\subsection{Mean flow results}

Detailed boundary-layer measurements were acquired at a series of $x$-locations. The profiles were integrated to obtain the displacement thicknesses, $\delta^{*}$. A linear curve fit was applied to $\delta^{* 2}$ versus $x$ and extrapolated to $\delta^{* 2}=0$ to get the virtual origin, $x_{0}\left(x_{0}=65.8 \mathrm{~mm}\right)$. The average value of the experimental shape factor was $\bar{H}=2.60 \pm 0.04$. The streamwise distance relative to the virtual origin, $x_{v}$, was used to compute the normalized wall-normal coordinate $\eta=\left(y / x_{v}\right) \cdot \operatorname{Re}_{x}^{1 / 2}$ where $R e_{x}$ is based on $x_{v}$. Excellent agreement was observed between the measured profiles and the Blasius profile. Transition Reynolds number $R e_{t r}$ measured on the smooth plate without controlled acoustic forcing was $R e_{t r} \approx 3.2 \times 10^{6}$. 


\subsection{Two-dimensional and oblique disturbances}

Results are presented and discussed for disturbances measured with 2-D and oblique surface waviness $\left(\psi_{w}=0^{\circ}, 15^{\circ}, 30^{\circ}\right.$, and $45^{\circ}$ where $\psi_{w}$ is the angle between roughness wavevector and streamwise direction). The Reynolds number at Branch I varied between $574<R_{I}<585\left(R=\sqrt{R e_{x}}\right)$. The T$S$ wavelength at Branch I, $\lambda_{t s, I}$, was computed using linear stability theory (LST). The wall roughness wavelength, $\lambda_{w}$, was selected to match $\lambda_{t s, I}$, the near resonance condition. Amplitudes of the measured O-S eigenfunctions, $u_{t s}$, for $\epsilon_{2} \cdot \Delta h_{2}$ are presented in Figure 1 (a) along with LST calculations. The appearance of a dual peak structure in the near-wall region that straddles the critical layer $(\eta \approx 1)$ was observed in the oblique eigenfunctions. For 2D disturbances, a single peak in the near-wall region below the critical layer was evident. As the disturbances became oblique, some residual of this peak remained with a smaller amplitude below the critical layer, and an apparent upward shift of the global maximum to a wall-normal location above the critical layer was observed. The amplitude of the apparent residual peak reduced with increased obliqueness. Figure 1 (b) shows a plot of $N$-factor versus Reynolds number along with the experimentally measured maximum T-S amplitudes, $\ln \left(u_{t s} / u_{a c}\right)$. Excellent agreement was observed between the measured and LST amplification curves for small oblique angles. The agreement became less attractive for the larger oblique angles, particularly near the location of Branch II. This may be a result of nonparallel effects not captured by LST.

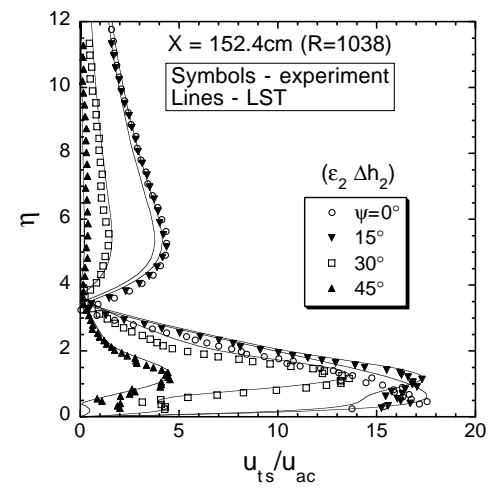

(a)

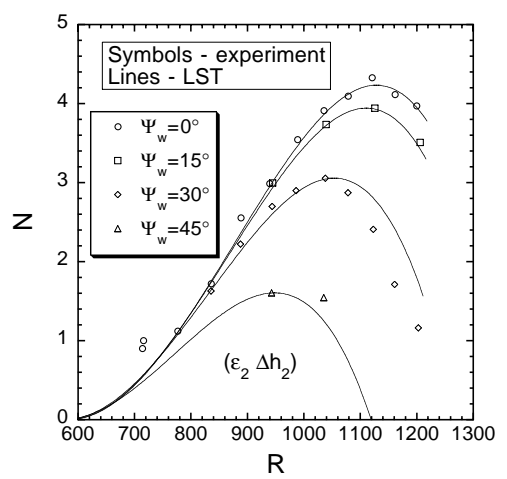

(b)

Fig. 1. Plots of (a) T-S amplitude profiles and (b) streamwise amplification data for various values of $\psi_{w}$.

The receptivity coefficients, $C$, were referenced to Branch I $\left(C=u_{t s, I} / u_{a c}\right)$. Linear receptivity was observed for all forcing combinations considered for the 2-D waviness (only one forcing combination $\left(\epsilon_{2} \cdot \Delta h_{2}\right)$ was examined for the oblique configurations). Figure 2 (a) shows a plot of measured $C$ versus $\psi_{w}$ along with theoretical data [2] $\left(F=56 \times 10^{-6}\right)$. Good agreement is observed, thereby providing experimental evidence of increased receptivity with 


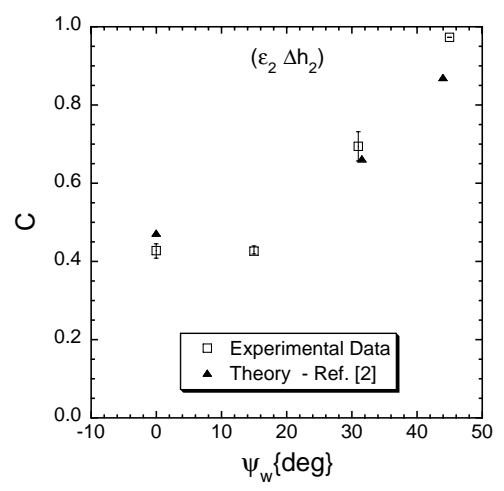

(a)



(b)

Fig. 2. (a) Receptivity coefficients versus $\psi_{w}$ and (b) receptivity coefficients versus detuning parameter.

increased wave obliqueness. The error bars denote the maximum plus/minus deviation from the mean receptivity values denoted by the symbols. Twodimensional receptivity coefficients compare favorably with other experimental data [4] $\left(F \approx 46 \times 10^{-6}\right)$. T-S wave kinematics were inferred from data obtained with short streamwise surveys $\left(\approx 1.5 \lambda_{t s}\right)$. The maximum percent difference between the measured and LST phase speeds was $5 \%$. Detuning was examined by varying $\lambda_{w}$ and measuring the T-S response. The detuning parameter was defined as $\sigma=\left(\alpha_{w}-\alpha_{t s, I}\right) / \alpha_{t s, I}$. Detuned receptivity coefficients for 2-D and oblique $\left(\psi_{w}=30^{\circ}\right)$ waviness are presented in Figure 2 (b). To account for finite wave speed $\left(\alpha_{a c} \neq 0\right)$, the detuning parameter $\sigma^{\prime}$ shifted the values of $\sigma$ by $\alpha_{a c} / \alpha_{t s, I}$. Good agreement is observed between the theoretical [2,3] and experimental data.

\subsection{Three-dimensional disturbances: oblique transition}

Oblique transition was initiated by generating a pair of oblique $\mathrm{O}-\mathrm{S}$ modes with the use of non-localized 3-D surface waviness (two intersecting oblique waviness patterns with $\psi_{w}= \pm 30^{\circ}$ ). A new spanwise coordinate, $\zeta$, was defined where $\zeta=0$ and $\zeta=1$ denote the centerline of the roughness pattern and the location one roughness spanwise wavelength $\left(\lambda_{w, z}=90.7 \mathrm{~mm}\right)$ away, respectively. Streamwise velocity measurements were acquired along the spanwise direction (typically over one $\lambda_{w, z}$ ) near wall-normal locations corresponding to the maximum amplitude of the 3 -D disturbance eigenfunction, $u_{t}$. Results are presented for two scenarios, one where the flow remained laminar $\left(\epsilon_{3} \cdot \Delta h_{1}\right)$ and one where laminar breakdown occurred $\left(\epsilon_{3} \cdot \Delta h_{3}\right)$. For $\epsilon_{3} \cdot \Delta h_{1}$, a peakvalley structure consistent with the roughness pattern was observed in the spanwise profile of $U$ which was very similar to classical results [8], although generated by a somewhat different mechanism. The peaks and valleys were defined as regions with large $u_{t}$ and small $u_{t}$, respectively. The spanwise distance between adjacent peaks was $\lambda_{w, z} / 2$. The distorted base flow was produced by 
the stationary vortex system $(0, \pm 2)$ which resulted from the nonlinear interaction of the primary modes $(1, \pm 1)$. Significantly more three-dimensionality associated with the streaks was observed for $\epsilon_{3} \cdot \Delta h_{3}$. The mean velocity increased by as much as $75 \%$ in the valley regions. For $R \leq 967$, the spanwise distribution of $u_{t}$ was consistent with results obtained for $\epsilon_{3} \cdot \Delta h_{1}$ (see Figure 3). The spanwise disturbance distribution took on a different character at $R=991$. An upwelling of $u_{t}$ on both sides of the peaks was observed. Explosive growths in the value of $u_{t}$ at these spanwise locations were realized with $x$. The spanwise locations of the maximum value of $|d U / d \zeta|$ coincided with the locations of maximum $u_{t}$. The magnitude of $u_{t}$ also scaled with $|d U / d \zeta|$. This explosive instability appeared to be driven by $d U / d \zeta$. Similar finding were reported for oblique transition in plane Poiseuille flow [6].



Fig. 3. Root-mean-square narrow-band velocity at $f_{0}$ measured along spanwise coordinate $\zeta$ at various $x$-locations for $\epsilon_{3} \cdot \Delta h_{3}$. Note the change in the spanwise distribution of $\boldsymbol{u}_{t}$ with increasing $x$ (i.e. upwelling of $\boldsymbol{u}_{t}$ on sides of peaks).

The difference between the maximum and minimum value of $U$ in each spanwise profile was used as a measure of the streak amplitude. It was interesting to note that the streak amplitudes measured at the first streamwise position ( $R=838$ ), $21 \mathrm{~cm}$ downstream of the roughness samples, were the same for both forcing combinations. Since the stationary streamwise vortex system $(0,2)$ was responsible for the formation of the streaks, this suggested that the $(0,2)$ modes were of equal strengths for both forcing combinations. However, the subsequent streak amplitudes differed significantly downstream (by a factor of 2 just downstream at $R=943$ ), suggesting that nonlinear interactions of nonstationary boundary-layer disturbances were responsible for the difference in streak amplification rates assuming some threshold amplitude of the $(0, \pm 2)$ mode was satisfied. It was therefore instructive to examine the disturbance evolution in the frequency-wavenumber plane. Figure 4 presents results obtained for the two forcing combinations. Modal velocities are presented for (i) the primary oblique modes $-(1,1)-$, (ii) the first set of nonlinear interactions $-(0,0),(0,2),(2,0)$, and $(2,2)-$, and (iii) selected higher-order interactions - $(1,3),(3,1)$, and $(3,3)$. For $\epsilon_{3} \cdot \Delta h_{1}$, the modal data in part (a) of the figure indicate a dampening of all the significant nonstationary modes after some initial amplification. However, when laminar breakdown was present $\left(\epsilon_{3} \cdot \Delta h_{3}\right)$, all nonstationary modes were seen to amplify significantly as expected (see part 
(b) of the figure). The nonstationary mode $(1,1)$ at $R=838$ for $\epsilon_{3} \cdot \Delta h_{3}$ was marginally larger than that for $\epsilon_{3} \cdot \Delta h_{1}$. However, the same was not true for the nonstationary mode $(1,3)$ at $R=838$. This modal velocity for $\epsilon_{3} \cdot \Delta h_{3}$ was approximately a factor of 20 larger than that for $\epsilon_{3} \cdot \Delta h_{1}$. A nonlinear interaction of the $(1,1)$ mode with the $(1,3)$ mode is a possible path for the enhancement of the stationary $(0,2)$ mode. These results support the conjecture proposed earlier concerning the observed differences in the growth rates of the measured streak amplitudes with $\epsilon \cdot \Delta h$. Excellent qualitative agreement between the measured modal velocities and the simulation results [9] was observed.

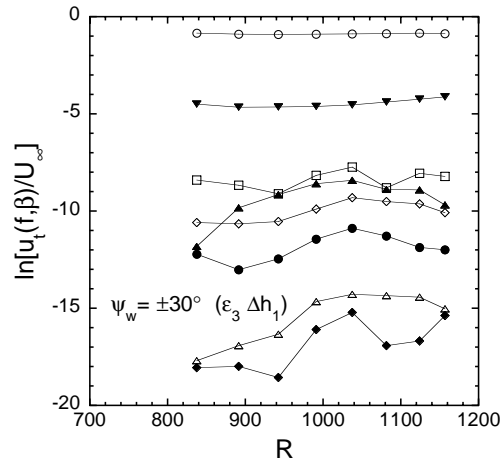

(a)

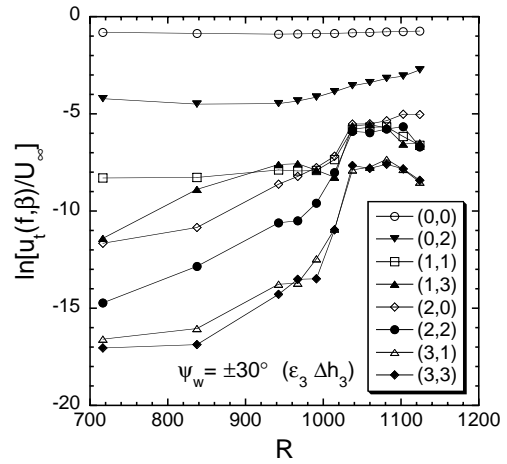

(b)

Fig. 4. Modal streamwise velocity distribution for (a) $\epsilon_{3} \cdot \Delta h_{1}$ and (b) $\epsilon_{3} \cdot \Delta h_{3}$. The mode designations given in the plot legends are $\left(f / f_{o}, \beta / \beta_{w}\right)$.

\section{References}

1. Goldstein, M. E. (1985), Scattering of acoustic waves into Tollmien-Schlichting waves by small streamwise variations in surface geometry. J. Fluid Mech., 154, $509-529$

2. Crouch, J. D. and Bertolotti F. P. (1992), Nonlocalized receptivity of boundary layers to three-dimensional disturbances. AIAA Paper 92-0740

3. Choudhari, M. (1993), Boundary-layer receptivity due to distributed surface imperfections of a deterministic or random nature. Theoret. Comput. Fluid Dynamics, 4, 101-117

4. Wiegel, M. and Wlezien, R. W. (1993) Acoustic receptivity of laminar boundary layers over wavy walls. AIAA Paper 93-3280

5. Schmid, P. J. and Henningson, D. S. (1992), A new mechanism for rapid transition involving a pair of oblique waves. Phys. Fluids A, 4, 1986-1989

6. Elofsson, P. A. and Alfredsson, P. H. (1998), An experimental study of oblique transition in plane Poiseuille flow. J. Fluid Mech., 358, 177-202

7. King R. A. (1999) Receptivity and Growth of Two- and Three-Dimensional Disturbances in a Blasius Boundary Layer. PhD Thesis, Massachusetts Institute of Technology, Massachusetts (to be submitted, November 1999)

8. Klebanoff, P. S., Tidstrom, K. D. and Sargent, L. M. (1962), The threedimensional nature of boundary layer instability. J. Fluid Mech., 12, 1-34

9. Joslin, R. D., Streett, C. L. and Chang, C.-L. (1993), Spatial direct numerical simulation of boundary-layer transition mechanisms: Validation of PSE theory. Theoret. Comput. Fluid Dynamics, 4, 271-288 